\title{
LOS HORTICULTORES de la Sabana Centro de Cundinamarca \\ no tienen una organización moderna
}

\section{ESUMEN}

¿Cómo hacer para que un grupo de cultivadores de hortalizas cambien de una estructura de subsistencia a organización. ¿En forma de conglomerado como una red de productores que garantice una oferta de calidad nacional e internacional?

En este artículo se resume la metodología aplicada y los principales resultados obtenidos, gracias a la aplicación de herramientas de prospectiva, así como análisis de modelos de redes económicas, teorías de dinámica empresarial, estudio de variables de entorno nacional $y$ de tendencias internacionales sobre los productos hortícolas y en una región seleccionada como fueron los municipios de la provincia de Sabana Centro en Cundinamarca.

\section{palabras clave}

Agronegocios, prospectiva, redes regionales de negocios, inversionistas estratégicos.
Por:

Alberto Villate París

$\mathbf{E}$ pasado mes de marzo el Grupo GENI -Grupo en Negocios Internacionales- presentó a Colciencias el informe final revisado del estudio de «Prospectiva de la cadena hortícola en la Sabana de Bogotá desde el lado de la oferta», que se desarrolló durante doce meses.

Este estudio se preguntaba cómo hacer para que un grupo de cultivadores de hortalizas cambien de una organización de subsistencia a una moderna, en forma de conglomerado o como una red de productores, para obtener una oferta de calidad nacional e internacional.

El estudio aplicó herramientas prospectivas, así como modelos de análisis de redes económicas, teorías de dinámica empresarial, estudios de variables de entorno

\footnotetext{
Alberto Villate París. Ingeniero de la Universidad Nacional de Colombia, con Maestría en Ingeniería de la Universidad de Boston, especialización en economía en la Universidad Católica de Chile y cursos de actualización en gerencia. Experto en planeación, y proyectos. Consultor en organización y regiones. Director de Planeación Distrital en la primera Administración de Mockus, y gerente de Fonade en la Administración Gaviria. Consultor con CIC geomática, Prospectiva S.A., econometría, tutoría empresarial S.A. Trabajó en la Comunidad Andina de Naciones, en el IFI, en la Universidad Nacional, Facultad de Ingeniería, y en el DNP. Redactor económico de Semana (1987) y Editor de Estrategia (1988-1990). Miembro del Grupo GENI - Grupo en Negocios Internacionales- de la Facultad de Ciencias Económicas y Administrativas de la Pontificia Universidad Javeriana, Grupo reconocido por Colciencias y del cual forman parte Juan M. Andrade, Hernando Otero, y José A. Pérez. Para este trabajo se contó con la asesoría de Fernando Nieto y de Pedro Amaya, prematuramente fallecido y a quien le rendimos homenaje. alberto.villate $a$ javeriana.edu.co
} 
nacional y de tendencias internacionales sobre los productos hortícolas en los municipios de la provincia de Sabana Centro, en Cundinamarca.

Para efectuar ese cambio se necesitan inversionistas estratégicos, empresarios con dinero y conocimientos del negocio, capitanes de empresa que arriesguen recursos para contratar a futuro la producción de hortalizas de calidad, lo cual implica financiación y controles en todos los eslabones de la cadena de suministro del negocio; hombres de negocio que generen la «destrucción creativa» de que habla Schumpeter.

El estudio combinó el enfoque teórico con la práctica y con los criterios de los empresarios para el análisis del entorno. La participación permanente del director de la Mesa de Logística del CARCE Consejo Asesor Regional de Comercio Exteriorde Bogotá -Cundinamarca y de miembros de la Mesa Agroindustrial del mismo CARCE fue valiosa para orientar el trabajo. Asimismo, la participación de más de treinta empresarios y expertos en el tema hortícola en los talleres de prospectiva fue determinante para construir la respuesta a la pregunta inicial y poder identificar las restricciones y las oportunidades para transformar una organización hortícola centrada en la subsistencia en una moderna comercial y de exportación'1.

En este artículo se resume la metodología aplicada y los principales resultados obtenidos.

\section{BSTRACT}

How to change the subsistence structure of a group of vegetable farmers into a real and modern organization? Maybe as a cluster of producers which guarantee the quality of the national and international demand. This paper describes the methodology and the results of a studio by means of prospective tools, analyses of economic models, dynamic in management theories and analyses of national and international variables upon vegetables products in Sabana Centro de Cundinamarca, a central region near the surroundings of Bogotá, Colombia

\section{KEY WORDS}

Agro-business, prospective, regional business clusters, strategic investment

\footnotetext{
1 La orientación permanente de Enrique Tafur, Director de la Mesa de Logística del CARCE y de Rafael Mariño, miembro de la Mesa Agroindustrial del CARCE se agradece sobremanera.
} 


\section{LA METODOLOGÍA APLICADA}

\subsection{Las 4P del conocimiento}

\section{Procesos}

El primer proceso de conocimiento fue seleccionar la zona de estudio para la producción de hortalizas con valor agregado. Para ello, se aplicaron los siguientes criterios:

- Disponibilidad, en la zona de fuentes hídricas adecuadas para cultivos limpios y saludables.

- Infraestructura de transporte, dada la cercanía al aeropuerto internacional de Bogotá.

- Infraestructura social, que se concreta en una tendencia regional a la asociación.

- Experiencia de los agricultores en el cultivo de hortalizas.

La Provincia de Sabana Centro de Cundinamarca fue elegida para este estudio. Aunque en términos de experiencia en el cultivo de hortalizas solo concentra el $13 \%$ de la producción de estos productos en la tierra fría del departamento (Sabana Occidente concentra $26 \%$, la provincia de Oriente $17 \%$ ) la Sabana Centro presenta la mayor diversidad pues cuenta con ocho tipos de cultivo que, en importancia según el número de hectáreas cultivadas en 2004, son: arveja, zanahoria, remolacha, brócoli, cilantro, espinaca, acelga, y coliflor.

Esta provincia tiene la mejor calidad de agua para riego de hortalizas, está cerca del aeropuerto internacional y presenta cinco cooperativas comercializadoras de hortalizas en funcionamiento, cuatro de ellas localizadas en Cota. En términos de población, el municipio más pequeño es Gachancipá y el más grande Zipaquirá, que es 15 veces mayor. Seis municipios dependen de la cuenca del río Bogotá, tres del río Frío, uno del Teusacá, otro del Chicú y otro más del Neusa. Los productos agrícolas son variados, así como la producción pecuaria y el número de establecimientos comerciales.

En un segundo momento se seleccionaron las herramientas de análisis. Se empezó por el nivel general de estudio del entorno, para analizar posteriormente la dinámica empresarial. Finalmente se estableció el nivel intermedio de variables de carácter regional, como se detalla más adelante.

El tercer proceso incluyó los talleres de prospectiva, partiendo de un listado de las variables clave para el análisis. Se convocaron dos talleres para construir la matriz de importancia y gobernabilidad, y la matriz de análisis estructural.

\section{Personas}

Una vez seleccionada la región piloto del estudio, se identificaron las relaciones entre los agentes de los eslabones de la cadena de valor tomando como referencia a los productores de hortalizas.

Los principales agentes en los vínculos hacia atrás son los productores y comercializadores de insumos de producción tales como: servicios de control biológico de plagas y enfermedades, alquiler de maquinaria agrícola, y provisión de insumos físicos como semillas y esquejes certificados, material vegetal, fertilizantes, pesticidas y otros agroquímicos. 
Hacia adelante, los principales eslabones de la cadena de valor son: los centros de post cosecha y servicios de selección, clasificación por calidades, refrigeración y codificación de barras.

En la articulación total de los eslabones de la cadena de valor encontramos a los agentes que proporcionan la logística y la organización para el manejo de los productos.

\section{Presiones}

La principal presión proviene de las redes comerciales regionales actuales, centradas en la atención del mercado interno. Estos canales de distribución, la mayoría con culturas comerciales tradicionales y en algunos casos modernos, como los canales de grandes superficies, generan tendencias, restricciones y presiones para el cambio. en este estudio se ha intentado identificar, desde el punto de vista de la gestión, el papel de estos elementos de organización social, sus fortalezas y debilidades.

En términos de competencia internacional, se analizó la importancia que tuvo para España el cambio en el sector agrícola debido a su participación en el esquema de integración europeo y a los retos de la globalización. Asimismo, se reseñaron algunos estudios sobre las experiencias de México dentro de NAFTA y se examinó, sobre los modelos de negocio, las oportunidades y los retos de la competencia hemisférica-ALCA, TLC, CAN, MERCOSURen el agro negocio de la horticultura.

\section{Productos}

El estudio ha tomado como punto de partida el análisis, basado en el Informe de «Exploración de mercados en la Ciudad de Miami (USA) y en la ciudad de Madrid (España) para 15 productos estratégicos del Departamento de Cundinamarca» dentro del programa de desarrollo del sector exportador no-tradicional del departamento de Cundinamarca presentado por la Corporación Colombia Internacional CCI en noviembre de 2004.

Allí se señalan las siguientes posibilidades de mercado para los productos seleccionados:

Miami: frutas: uchuva y pitahaya; hortalizas frescas: arveja y lechuga en posibilidad media.

Madrid:frutas: Uchuva, granadilla, maracuyá, tomate de árbol y pitahaya; hortalizas frescas: arveja y lechuga.

\subsection{Prospectiva}

La prospectiva es una forma de hacer previsión acerca de cuales son los elementos de organización y las tecnologías claves para alcanzar el desarrollo. Los métodos de la prospectiva global y tecnológica estuvieron marcados por cuatro corrientes desde los años 70. Godet (1999, p. 6 y ss) citando a Philippart (1986), las cataloga en postindustrial, neomalthusiana, de ciclos largos y de bifurcaciones y caos.

Son varios los autores en cada corriente. Por ejemplo, en la postindustrial se recuerda a Toffler, quien en Choque del Futuro (1971) crea la noción de pronóstico tecnológico. Dentro de la segunda corriente Godet plantea los modelos de dinámica de sistemas de $\mathrm{J}$. Forrester y los estudios de Meadows de 1972 para el Club de Roma sobre los «límites del crecimiento». Kondratiev es el máximo exponente de los ciclos largos, junto con la escuela de la regulación francesa. Las bifurcaciones y el caos comprende desarrollos de Poincaré realizados por Lorenz (1961), Prigogine y Stengers (1979), Atlan, Prigoginey 
y Gleick. Hay palabras que hacen soñar, como dice Godet: los fractales, los atractores extraños, las cascadas de las bifurcaciones, el efecto mariposa, entre otros, o que inspiran miedo como: ruido, desorden, catástrofe, caos.

Según el Centro de Prospectiva Tecnológica de la Unión Europea, existen tres formas de denominar la prospectiva:

- Pronóstico tecnológico (Technology Forecasting). Estudia las previsiones probabilísticas de desarrollos tecnológicos futuros.

- Evaluación tecnológica (Technology Assessment). Evalúa los impactos futuros sobre la economía y la sociedad, a partir de tecnologías nuevas conocidas.

- Prospectiva Tecnológica (Technology Foresight). Analiza las prioridades científicas y tecnológicas actuales, a la luz de proyecciones hipotéticas hacia el futuro, conforme los desarrollos económicos, sociales y tecnológicos.

A partir de los años noventa, varias empresas, sobre todo europeas, iniciaron ambiciosos programas de prospectiva tecnológica como base para diseñar estrategias. Estas empresas utilizaron varios instrumentos tales como:

- Método de exploración del entorno. Se da en la etapa inicial de un estudio de prospectiva y consiste en la identificación de un amplio rango de factores relevantes al tema de interés, y la recolección y el análisis de la información sobre esos factores.

- Análisis de tendencias. Extrapolación de datos históricos donde hay una base para asumir que la tendencia seguirá un comportamiento establecido.
- Análisis estructural. Desarrollo de aplicaciones prácticas que permiten descubrir y analizar las interrelaciones entre fenómenos, objetos o conceptos para así usar los resultados en la construcción de nuevos sistemas sociales, económicos y políticos.

- Árboles de pertinencia. Herramienta normativa basada en el análisis de sistemas, que muestra los posibles caminos a seguir hacia un objetivo.

- Encuestas delphi. Desarrolladas por la RAND Corporation, son un cuestionario en el que el tema se somete como hipótesis de futuro a la opinión de los expertos. Una vez analizada la respuesta obtenida es enviada de nuevo a consideración de los que han respondido para que en una segunda ronda puedan variar su opinión en función de los resultados alcanzados. El proceso se repite hasta alcanzar un grado de acuerdo suficiente. Se trata de conseguir el mayor consenso posible en la respuesta con base en la calidad del juicio del grupo cuya opinión es considerada igual o mejor que las opiniones individuales.

- Escenarios. Construcción de un número de visiones internamente consistentes de futuros posibles, combinando la información disponible y las posibilidades de futuro, expresadas como una narración.

- Matriz de impacto cruzado. Su lógica básica subyacente consiste en hacer una exploración del futuro sobre la base de una serie de eventos que pueden o no ocurrir dentro de un horizonte temporal considerado. En tal sentido evento se refiere aquí a una hipótesis que puede o no ser cierta, según que tal evento ocurra o no en el marco temporal analizado. 
- Método de probabilidad de Bayes. Aplicación de las fórmulas derivadas del Teorema de Bayes a la determinación de las Ilamadas probabilidades revisadas, asociadas a un conjunto dado de hipótesis (escenarios factibles de presentarse) mutuamente excluyentes, como consecuencia de las evidencias (hechos) observados.

- Método de análisis de jerarquía de procesos. Técnica focalizada fundamentalmente como herramienta de apoyo a la toma de decisiones.

- Método de pensamiento complejo. Tiende a superar los problemas de la predicción, puesto que las partes del sistema no producen comportamientos lineales. En los sistemas no lineales se manifiestan cambios tales que en uno o dos parámetros ante un pequeño cambio en uno de ellos puede presentarse un gran cambio en todo el sistema.

\subsection{Organización empresarial}

\section{La colaboración entre empresas es una forma de organización empresarial}

La esencia de la colaboración entre empresas de la cadena (de suministro o de la cadena de contratos es el cumplimiento de las especificaciones y los costos.

La colaboración entre empresas surge cuando dos o más empresas voluntariamente acuerdan integrar recursos humanos, financieros 0 técnicos en un esfuerzo por crear un nuevo modelo de negocios más eficiente, más efectivo y más relevante. Cualquier relación que involucre compras, ventas o sub-contratación de funciones o de procesos puede ser reestructurada como colaboración entre empresas adecuando su mecanismo apropiado de gestión. A través de la colaboración entre empresas, las empresas que voluntariamente participan crean políticas comunes e integran procesos operacionales para eliminar duplicaciones y redundancias no productivas en la búsqueda de una máxima productividad.

La estructura de gestión de la colaboración entre empresas cambia de una organización bajo comando y control, a un marco de acuerdos mutuamente prescritos sobre la base de reglas de operación comunes.

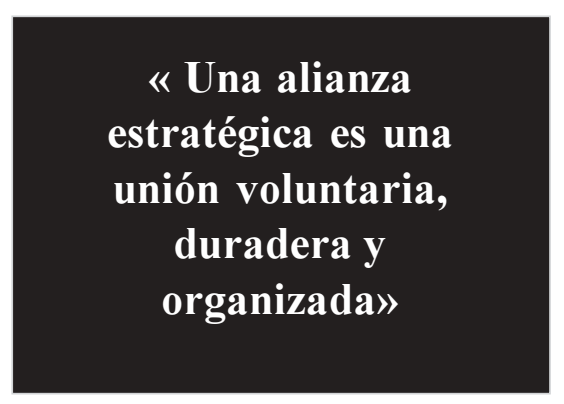

\section{Las alianzas estratégicas como herramientas de colaboración entre empresas}

Una alianza estratégica es una unión voluntaria, duradera y organizada de personas o empresas que ponen sus fuerzas en común para lograr ventajas especiales que les permiten estar en mejor situación competitiva. Algunas ventajas que se pueden conseguir de ellas son:

- Escala: para mejorar la capacidad de negociación y obtener descuentos.

- Financiamiento: para tener una mayor probabilidad de acceder a créditos o aumentar los avales. 
- Asesoría: disminuye costos fijos y permite el acceso a servicios especializados que como el marketing son inaccesibles en forma individual.

- Capacitación: posibilita contratar expertos que dicten cursos o asumir colectivamente los gastos de enviar a alguien a seminarios o talleres.

- Promoción conjunta: permite reducir costos y mejorar impactos.

- Gestión: una gerencia común permite rebajar costos, realizar promociones, concursos y publicidad, manejar tarjetas de descuento propias de la asociación.

- Transporte: reduce costos y tiempos.

- Integración vertical: es posible acortar y hacer eficiente la cadena de valor o de abastecimiento, según el concepto aplicado.

La forma jurídica de una Alianza Estratégica. El tipo de forma jurídica para conformar una alianza estratégica depende de:

- La intención o no de obtener lucro.

- Los objetivos del grupo a corto y largo plazo.

- El tipo de responsabilidad que se pretende asumir como integrante del grupo.

- El número de personas que va a constituir el grupo.

- La capacidad económica de los integrantes.

- La homogeneidad de los integrantes.

- La prestación de servicios a terceros o no.

- La necesidad de contratar personal asalariado o no.

- La voluntad para la libre admisión de nuevos participantes al grupo.

- El tipo de transmisión de la participación de cada integrante al grupo.

- El tamaño del mercado.
Las nuevas formas jurídicas de asociación empresarial:

- No requieren de personería jurídica, salvo para la declaración de impuestos.

- No prevén obligaciones y derechos adicionales a los de cada empresa que los conforma.

- No pierden su individualidad económica y legal.

- Se unen con una finalidad de cooperación, no para realizar una actividad en común sino para mejorar el funcionamiento de las agrupadas, para facilitar o desarrollar fases de la actividad de sus miembros, para perfeccionar o incrementar el resultado de tales actividades.

- Deben constituir un fondo común operativo que se mantendrá hasta la vigencia del contrato.

- Deben designar a una o más personas que dirijan y administren la agrupación y que los represente y actúe en nombre de la alianza.

\subsection{Redes: seis enfoques teóricos sobre las formas de asociación empresarial}

En el estudio se identificaron seis enfoques complementarios: económico, de confianza, capital social, enfoque sociológico, geográfico $y$ de negocios.

El enfoque económico. Según Grandori (1999), concibe y define las redes y las formas de coordinación empresarial como "un conjunto de empresas, generalmente caracterizadas por diferentes preferencias y recursos, coordinadas a través de una mezcla de mecanismos no limitados a precios, resultados y regulaciones".

La construcción de confianza. Autores como Fukuyama (1995) y Kramer y Tyler (1996), responden a algunas preguntas ligadas a la 
dinámica de la confianza en las organizaciones: ¿Ha decaído la confianza? ¿Cómo puede reconstruirse? ¿Qué tipos de colaboración regional existen? ¿Es durable la tendencia a moverse lejos de formas jerárquicas hacia agrupamientos flexibles alrededor de proyectos?

La vertiente de capital social ha analizado desde el estudio pionero de Putnam et. al. (1993) en Italia hasta los recientes trabajos de Putnam (2002) sobre la evolución del concepto en la sociedad contemporánea.

El enfoque de la geografía tiene su más interesante exponente en Milton Santos (1995), quien define el espacio como el conjunto indisociable de sistemas de objetos naturales o fabricados y de sistemas de acciones deliberadas. Examina las transformaciones actuales del espacio geográfico a partir de tres elementos constitutivos de la época de la globalización. El espacio se vuelve más diversificado y heterogéneo. A la división tradicional en regiones se agrega otra, producto de los vectores de modernidad y regulación.

La nueva sociología económica rescata elementos de los distritos industriales de Marshall, enmarcados en la especialización flexible, que hacen referencia a una lógica de intercambios mercantiles orientada hacia la cooperación entre unidades productivas, que se traduce en redes de empresas e implica el reconocimiento a la existencia e influencia de factores culturales y sociales locales.

Las redes regionales de negocios, según Casson (2000), involucran vínculos basados en comunicaciones y flujos de información de alta calidad. Los agentes racionales se unen a una red porque saben que pueden beneficiarse de menores costos de transacción, cuyos mejores ejemplos se relacionan con la coordinación del flujo de productos. La esencia de una red es el flujo de información altamente confiable.
Casson concluye que el proceso de vinculación se hace por intermedio de lo que se llaman las redes, que son vectores de modernidad y también de entropía. Hay buenas redes y malas redes desde el punto de vista del desarrollo económico regional. Las primeras son abiertas, transparentes y empresariales e involucran la provisión de bienes públicos al sector. Las redes malas son cerradas y opacas, e involucran a políticos o elites antiemprendedoras, buscadores de renta, lobystas, protectores de empresas regionales débiles contra la competencia externa.

\subsection{Entorno}

\section{El sector hortícola colombiano presenta un gran potencial}

Los cultivos de hortalizas, según el Plan de Modernización de la Horticultura Colombiana elaborado por Corpoica, son generadoras de mano de obra, pero los elevados costos de producción, por el uso de pesticidas, de fertilizantes químicos u orgánicos y la baja eficiencia de la mano de obra utilizada en las prácticas de manejo, limitan el crecimiento del sector, siendo este uno de los sistemas menos eficientes en el manejo de recursos, lo que va en contravía de los criterios de competitividad y sostenibilidad que demanda la organización actual de mercados.

\section{El entorno internacional}

Desde un punto de vista prospectivo se identificó, mediante análisis de tendencias, los factores que inciden en el crecimiento del mercado hortícola en el mundo.

En primer lugar, están los cambios en los hábitos alimenticios de la población debidos a una mayor preocupación por el cuidado de 
la salud. La orientación hacia fuentes de energía bajas en grasa y con alto contenido en fibra, favorece el consumo de los vegetales frescos y procesados.

En segundo lugar, hay una mayor demanda por productos llamados «de conveniencia» 0 listos para consumir, sin tener que pelar, lavar, desinfectar o hervir, en el caso de las hortalizas.

En tercer lugar, el proceso de concentración e internacionalización de la distribución de alimentos se da dentro de las llamadas cadenas comerciales globales que modifican los hábitos de compra y de consumo de la población que con un mayor poder adquisitivo y con mayores preocupaciones para el buen uso de su tiempo libre fuera del trabajo exigen cada vez mayor calidad en productos y servicios.

En cuarto lugar, los distribuidores y los grandes almacenes están pasando de atender el mercado étnico reducido y poco exigente, así como el mercado de «fuera de temporada»a mantener una oferta permanente.

En quinto lugar hay nuevos productos preparados (prelavados, cortados), empacados (empaque conjunto de alimentos complementarios), presentados (productos exóticos con nuevas recetas).

\section{La reciente transformación agrícola de España deja enseñanzas}

La discusión en España giró, en los años 90, en torno a la forma de transición de la economía agraria tradicional a una nueva economía rural de carácter multifuncional y plurisectorial.

Este cambio no fue el resultado de una elección de los protagonistas, sino más bien de la lógica del proceso de modernización y desarrollo comunitario europeo, en donde se funden los conceptos de desarrollo agrario y desarrollo rural que, junto con la calidad, la variedad y la marca, son los pilares fundamentales de la PAC -Política Agrícola Común-europea.

Son dos los motores del cambio rural europeo: la capacidad endógena del mundo rural y de la comarca, que se considera como el factor más decisivo de estos procesos de modernización y de cambio, por un lado, y las políticas públicas, por el otro.

Algunas tendencias observadas en España son las siguientes:

- Una mayor relación con el resto de los sectores productivos, lo cual ha llevado a desarrollar el concepto de «complejo agroalimentario».

- La subcontratación de un número creciente de funciones

- Un incremento sustancial de ayudas gubernamentales o de la comunidad, como consecuencia de la integración de España en la Unión Europea.

- La tecnificación de las explotaciones, la generalización de la mecanización y el desarrollo de sistemas productivos muy intensivos en capital en un número mayor de productos.

- Un creciente proceso de organización interna en la fase de producción, que se traduce en una proporción cada vez mayor de agricultores dispuestos a formar redes de productores, cooperativas, alianzas y diversas formas asociativas. 


\section{RESULTADOS}

\subsection{Aplicación de las herramientas de prospectiva}

Se aplicaron cinco herramientas de prospectiva: exploración del entorno, análisis de tendencias, análisis morfológico, matriz de importancia y gobernabilidad y matriz de análisis estructural.
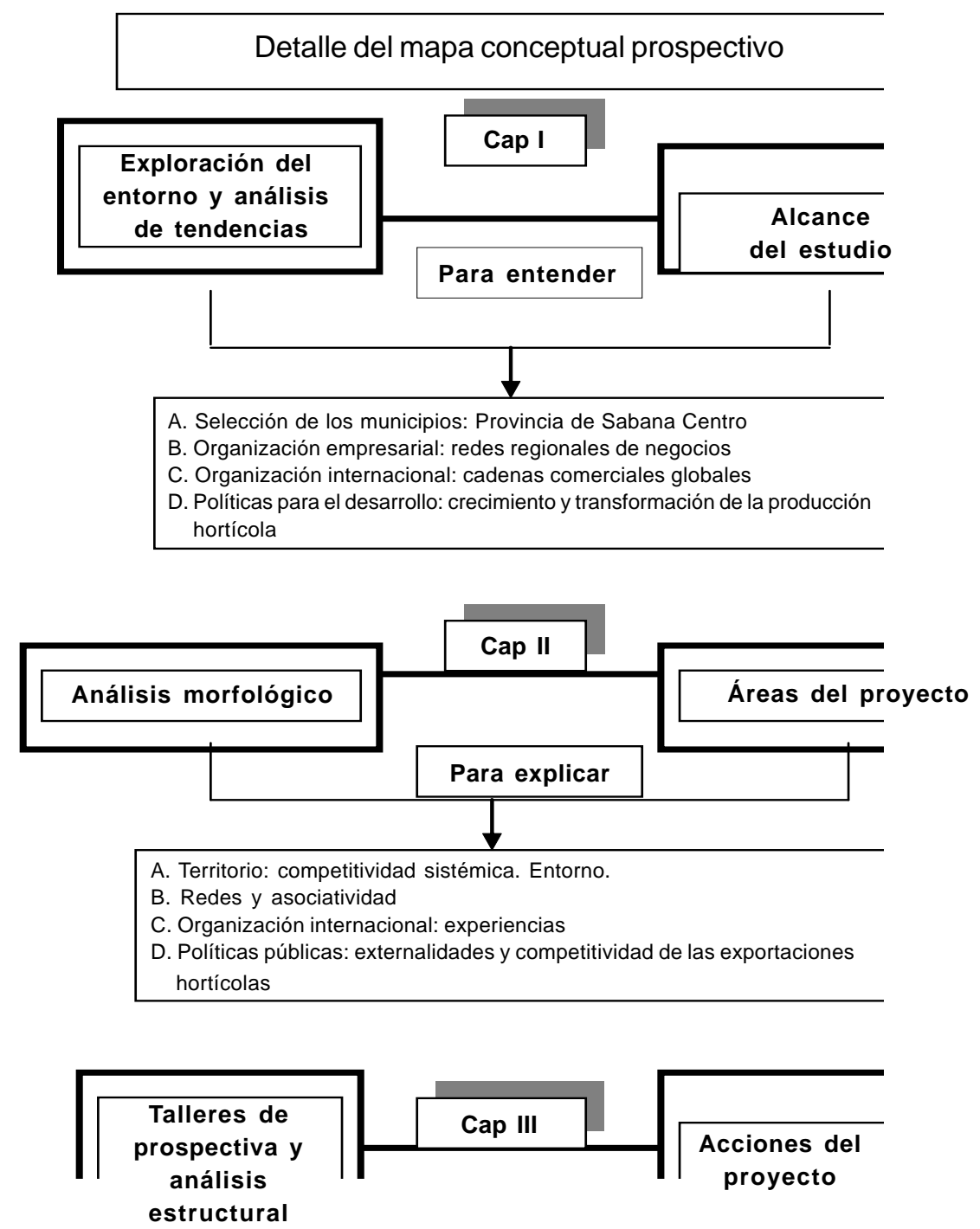

Para desarrollar 
En el siguiente cuadro se pueden apreciar en detalle las herramientas de prospectiva aplicadas:

\subsection{Selección de las variables clave}

Después de la discusión técnica sobre los instrumentos de prospectiva aplicados en este trabajo, se procedió a la identificación de las variables clave para la discusión en los talleres.

El proceso empezó identificando las principales variables de prospectiva al año 2025 que habían elaborado varios grupos de pensamiento, siendo el más importante el de la $\mathrm{CCl}$-Corporación Colombia Internacional-y el documento Colombia 2019 del DNP (2005).

Este resultado se discutió con los miembros del grupo de trabajo, y se acordó bajar el nivel de abstracción del contexto nacional, al nivel de empresa, aplicando los modelos planteados de Cinco Fuerzas y de Cadena de Valor Porter (1980, 1990), y las categorías de Jarillo (2003). En una nueva discusión con los asesores del CARCE, se llegó a la conclusión de complementar este ejercicio con otro marco teórico, más ligado al mercado y sus necesidades, como el planteado por Kaplan y Norton (2001).

En este proceso de retroalimentación, se cuestionó la utilidad de centrar la discusión con los empresarios sobre aspectos micro empresariales, a la luz de cada modelo de negocio, lo cual no permitiría obtener conclusiones de conjunto, que es lo buscado en este estudio. Se optó por tanto por analizar variables del negocio hortícola en el nivel de competitividad de las regiones o nivel mesoeconómico, intermedio entre la perspectiva nacional y la dinámica empresarial.
Las siguientes son las variables de competitividad regional resultantes de este proceso de discusión:

\section{Condiciones de la demanda externa}

- Falta de empresarios con vínculos en los mercados externos.

- Escasa presencia en mercados y nichos o segmentos calificados.

- Excesiva competencia en agricultura de productos con alto valor agregado.

- Excesiva regulación de protección al consumidor (sanitarias, empaque).

\section{Contexto de la empresa}

- Logística pobre, tanto en calidad como en cantidad

- Organización: poca experiencia en subcontratación y en integración.

- Competencia desleal. Informalidad. Abusos del comprador.

- Información empresarial: uso mínimo y dificultad de acceso a Internet y otras fuentes.

- Falta de incentivos y políticas para que las empresas hagan alianzas y atraigan inversión.

\section{Condiciones de la tecnología}

- Baja capacidad de producción agrícola regional de productos con alto valor agregado.

- Escaso desarrollo en: biotecnologías, paquetes tecnológicos, maquinaria y control de plagas y enfermedades.

- Falta de asistencia técnica en mejores prácticas.

- Dificultad para conciliar los intereses del productor con los requisitos del comprador.

- Reducida inversión pública y privada en ciencia, tecnología e innovación. 


\section{Condiciones de los factores}

- Escasez de agua apta para riego.

- Altos costos de transporte, comunicaciones y servicios públicos.

- Falta de seguridad jurídica, personal y física.

- Clientelismo para asignar los recursos públicos regionales.

- Corrupción, que eleva los costos de transacción.

- Falta de inversión privada en acciones, capital de riesgo, fondos cerrados de inversión y banca de inversión.

- Difícil acceso al crédito dirigido y altos costos comerciales.

- Falta de formación en gerencia.

- Desconfianza entre actores o falta de capital social.

- Falta de mano de obra calificada.

\subsection{Talleres de prospectiva para construir matrices IGO}

Este taller fue diseñado para un grupo de treinta participantes con una experiencia común -la producción agrícola-y que deseaban reflexionar sobre los cambios posibles y deseables inducidos por la competencia externa.

En un primer momento se expuso el documento de trabajo sobre prospectiva con el fin de analizar las adaptaciones que las empresas y las organizaciones deben realizar frente a los cambios que motivan un proceso de asociatividad flexible en redes.

Posteriormente se expusieron las 24 variables, teniendo en mente que se trataba de identificar tanto las principales restricciones que dificultan la asociatividad empresarial, (muy importantes $=5$, medianamente $=3$, poco $=1$ ) como el grado de control o dominio que se cree tener sobre cada variable (alto $=5$; medio $=3$; bajo $=1$ ) .

Para construir la Matriz IGO, cada uno estableció, por separado, sus propias prioridades (alta $=5$, media $=3$, baja $=1$ ) tanto en importancia como en gobernabilidad o control y se compartieron más tarde en la sesión plenaria para validar y ajustar.

El ejercicio individual y colectivo tenía cuatro opciones de resultados:

A. Problemas críticos e importantes, retos de futuro, que no se dominan actualmente.

B. Problemas importantes, que se estima están bien controlados.

C. Problemas poco importantes, que no se dominan, lo cual no es grave, pues son puntos débiles.

D. Problemas poco importantes que se controlan. Es sin duda de aquello de lo que se habla demasiado.

Al final se obtuvieron los siguientes resultados:

Variables de la opción A. Problemas críticos que son importantes, que no se dominan actualmente. Estos son los siguientes (los números entre paréntesis corresponden a las variables identificadas en el acápite anterior):

- Falta de empresarios con nexos en mercados externos.

- (7) Abusos del comprador.

- (8) Pobre información empresarial.

- (9) Falta de incentivos para atraer inversión y hacer alianzas.

- Falta de asistencia técnica en mejores prácticas.

- (14) Limitada inversión en ciencia y tecnología.

- (15) Falta de agua apta para riego.

- Altos costos de transporte, comunicaciones y logística pobre. 
- (20) Falta de inversión privada.

- (21) Difícil acceso al crédito dirigido.

- (23) Desconfianza entre actores o falta de capital social.

En la opción B. Problemas importantes, pero controlados, se encuentran las siguientes variables:

- Logística pobre, tanto en calidad como en cantidad.

- Organización: poca experiencia en subcontratación e integración.

- Escaso desarrollo tecnológico (biotecnología, paquetes tecnológicos, maquinaria, control de plagas y enfermedades).

- (17) Falta de seguridad jurídica, personal y física.

En la opción C. Problemas poco importantes, que no se dominan. Lo cual no es grave, pues son puntos débiles.

- Escasa presencia en los mercados y en los nichos o segmentos calificados.

- Excesiva competencia en agricultura de productos con alto valor agregado.

- Excesivas regulaciones de protección al consumidor (sanitarias, empaque).

- Amiguismo para asignar los recursos públicos regionales.

- Corrupción, que eleva los costos de transacción.

En la opción D. Problemas, poco importantes, que se controlan. Es sin duda, de aquello de lo que se habla demasiado. Estos son:

- Muy baja capacidad de producción regional agrícola de productos de alto valor agregado.

- Dificultad para conciliar los intereses del productor con los requisitos del comprador.

- Falta de formación en gerencia.

- Falta de mano de obra calificada.
En el cuadro de la página siguiente se puede apreciar el resultado de la discusión y el análisis, en términos de prioridades y grado de control estimado.

\subsection{Matriz de análisis estructural}

Godet (1999) señala que la técnica de análisis estructural se basa en la teoría de los sistemas, y utiliza herramientas del cálculo matricial. Enfatiza en que la estructura del sistema, es decir la red de relaciones entre estos elementos, es esencial para comprender su evolución puesto que esa estructura conserva cierta permanencia.

El análisis estructural pone de relieve estas relaciones entre las variables en forma cualitativa. Describe un sistema mediante una matriz que interconecta todos los componentes del sistema. El manejo de las matrices permite identificar las variables esenciales.

\section{Bases para construir la matriz de relaciones}

Antes de concluir que existe una relación entre dos variables, los participantes del taller respondieron sistemáticamente tres preguntas:

- Ejerce la variable i una acción efectiva sobre la variable j, o la relación será más bien de j hacia i.

- Esta variable i ejerce una acción sobre j o existe más bien una colinealidad, es decir, que una tercera variables $\mathrm{k}$ actúa sobre i y j.

- La relación entre i y j es directa, o más bien se realiza a través de otra variables $r$ de las incluidas en la lista. 


\section{Matríz de importancia y dominio}

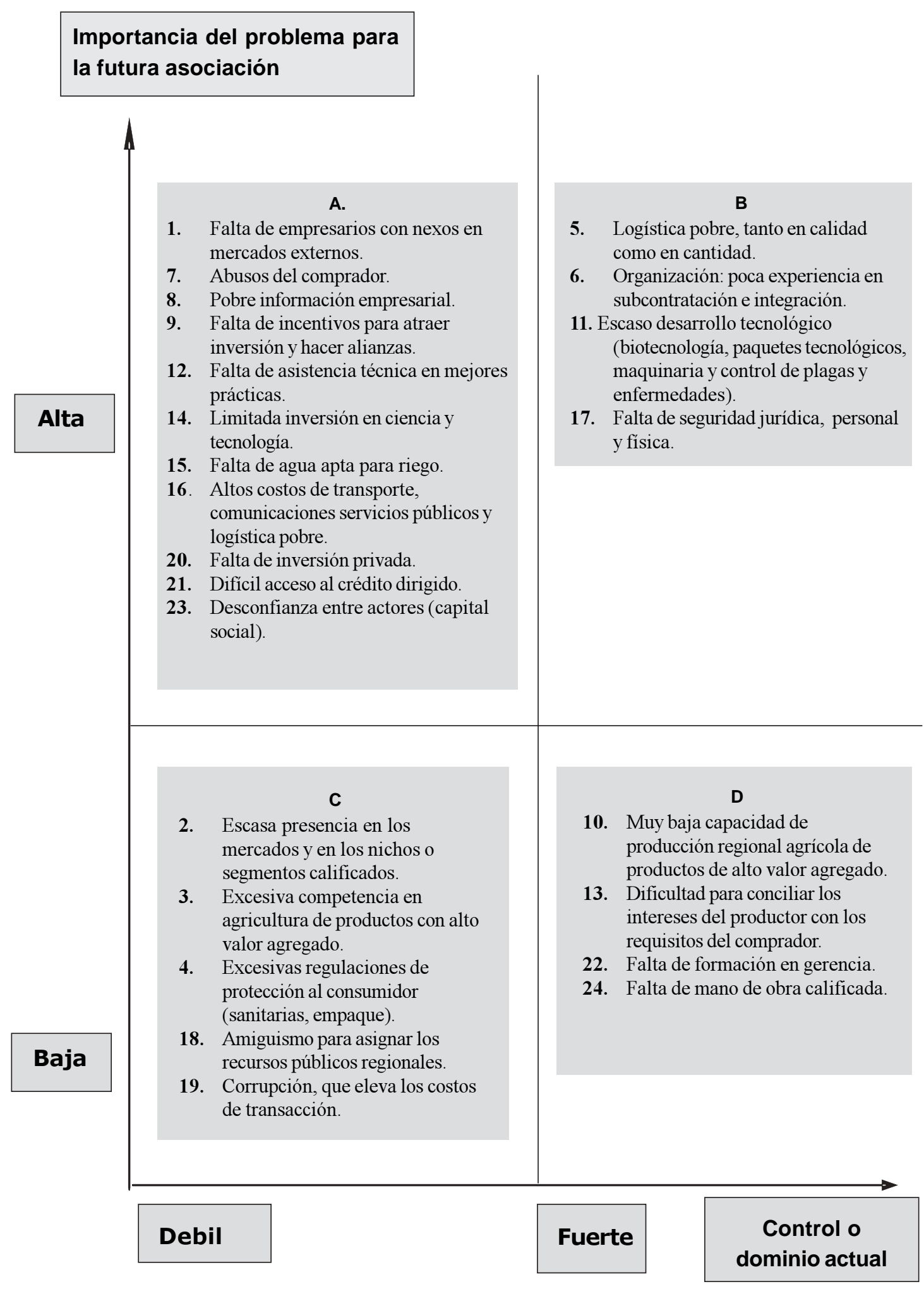


Este procedimiento de preguntas sistemáticas permitió evitar errores que se podrían haber cometido al llenar la matriz. La resolución de la matriz es tanto cualitativa (existencia o no de las relaciones) como cuantitativa. Se distinguen varios niveles de intensidad en las relaciones directas: fuertes (5), medias (3), débiles (1).

\section{Calificaciones:motricidad, dependencia, variables directas}

Se trata de hacer evidentes las variables más motrices y las más dependientes, como las llama Godet (1999). Las variables motrices son aquellas cuya evolución condiciona más el sistema.
Este plano de motricidad y dependencia puede dividirse en cuatro sectores:

- Variables muy motrices y poco dependientes: son las variables explicativas que condicionan el resto del sistema o zona de poder.

- Variables a la vez muy motrices y muy dependientes: son las variables de enlace, inestables por naturaleza. Es la zona de conflicto. Cualquier acción sobre estas variables repercutirá sobre las otras y tendrá un efecto bumerang sobre ellas mismas que amplificará o desactivará el impulso inicial.

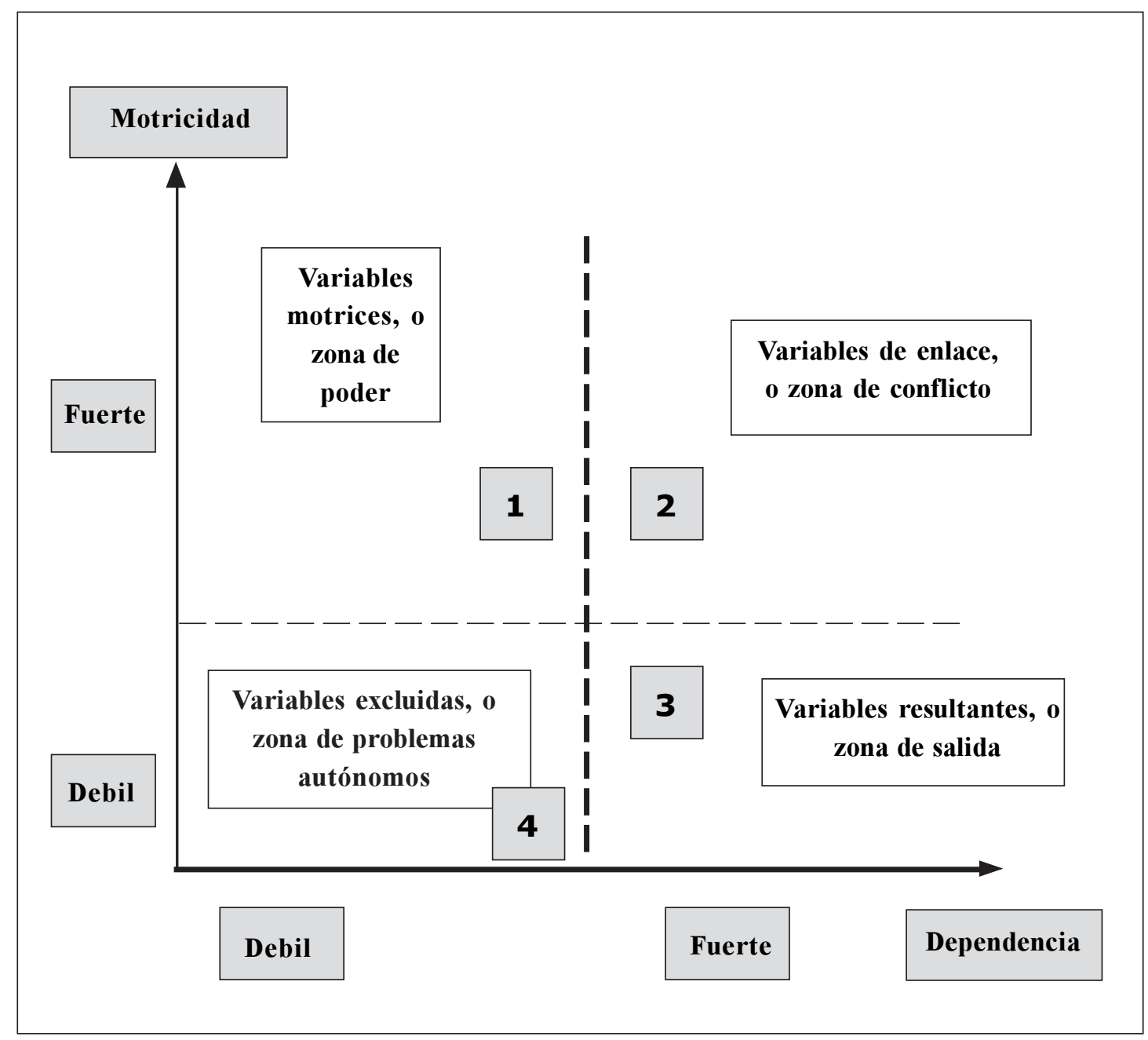


- Variables poco motrices y muy dependientes: son las variables resultantes cuya evolución se explica por la dinámica de las variables de los sectores 1 y 2 . Es la zona de salida.

- Variables poco motrices y poco dependientes: constituyen tendencias fuertes o factores relativamente autónomos. No son determinantes hacia el futuro. Pueden ser excluidas del análisis. Es la zona de problemas autónomos.

\section{Resultados de la matriz de relaciones directas}

Los participantes, empresarios y representantes de la sociedad civil, analizaron cada relación por separado y los resultados obtenidos fueron luego consolidados. Se discutieron aquellas variables que presentaron una dispersión extrema, para aclarar conceptos. Se llegó a acuerdos después de escuchar los argumentos y explicaciones de los participantes.
Los resultados finales, en números promedio, son los siguientes, se recuerda que 1 es bajo y 5 es alto:

En la zona de poder (alta motricidad y baja dependencia) se encuentran los siguientes problemas:

- Pobre información empresarial.

- Falta de asistencia técnica en mejores prácticas.

- Desconfianza entre actores o bajo capital social.

En la zona de conflicto (alta motricidad y alta dependencia) están:

- Falta de incentivos para atraer inversión y hacer alianzas.

- Limitada inversión en ciencia y tecnología.

- Falta de inversión privada.

- Difícil acceso al crédito dirigido.

En la zona de salida (alta dependencia y baja motricidad) está:

\section{Análisis estructural}

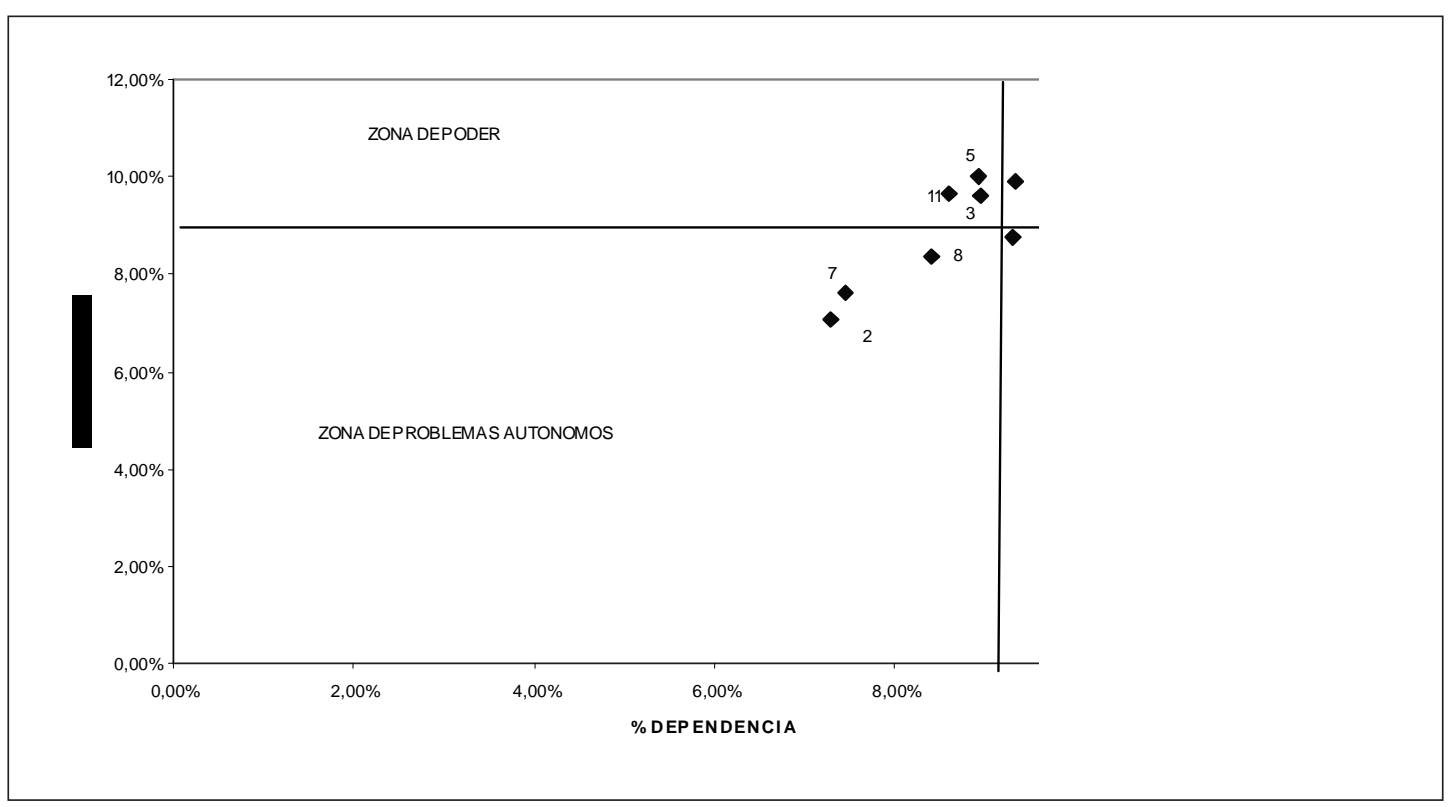


- Falta de empresarios con nexos en mercados externos

En la zona de problemas autónomos (baja motricidad y baja dependencia) están:

- Abuso del comprador

- Falta de agua apta para riego.

- Altos costos de transporte, comunicaciones, servicios públicos y logística pobre.

Este resultado fue revalidado una semana más tarde en Zipaquirá con trece actores de la cadena de valor hortícola, en la sede de la Cámara de Comercio de Bogotá.

\section{Calificaciones: motricidad, dependencia, variables indirectas}

Además de las relaciones directas entre variables, Godet (1999) señala que existen relaciones indirectas entre ellas, mediante cadenas de influencia y los llamados técnicamente «bucles de reacción» o de retroalimentación. Una matriz que incluya una docena de variables directas puede comprender cientos de interacciones indirectas.

En el gráfico siguiente se esquematiza este concepto:

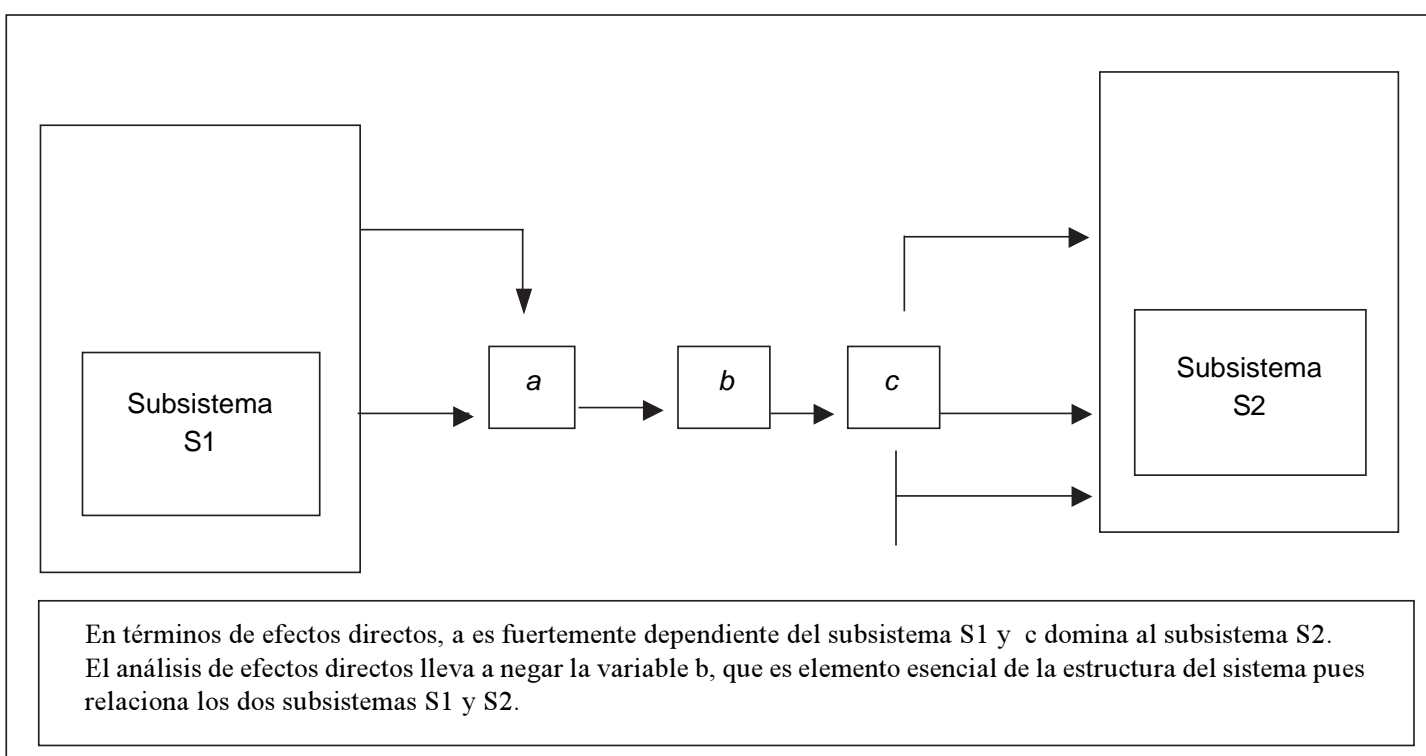

La multiplicación matricial, aplicada a la matriz de análisis estructural antes descrita, permitió estudiar la difusión de los impactos por los caminos y bucles de reacción y por consiguiente jerarquizar las variables tanto por orden de motricidad (teniendo en cuenta el número de caminos y bucles salidos de cada variable) como de dependencia, o número de caminos y bucles que llegan a cada variable. Si la variable i influye directamente sobre la variable $\mathrm{k}$, y si k influye directamente sobre la variable j, resulta el siguiente esquema: 


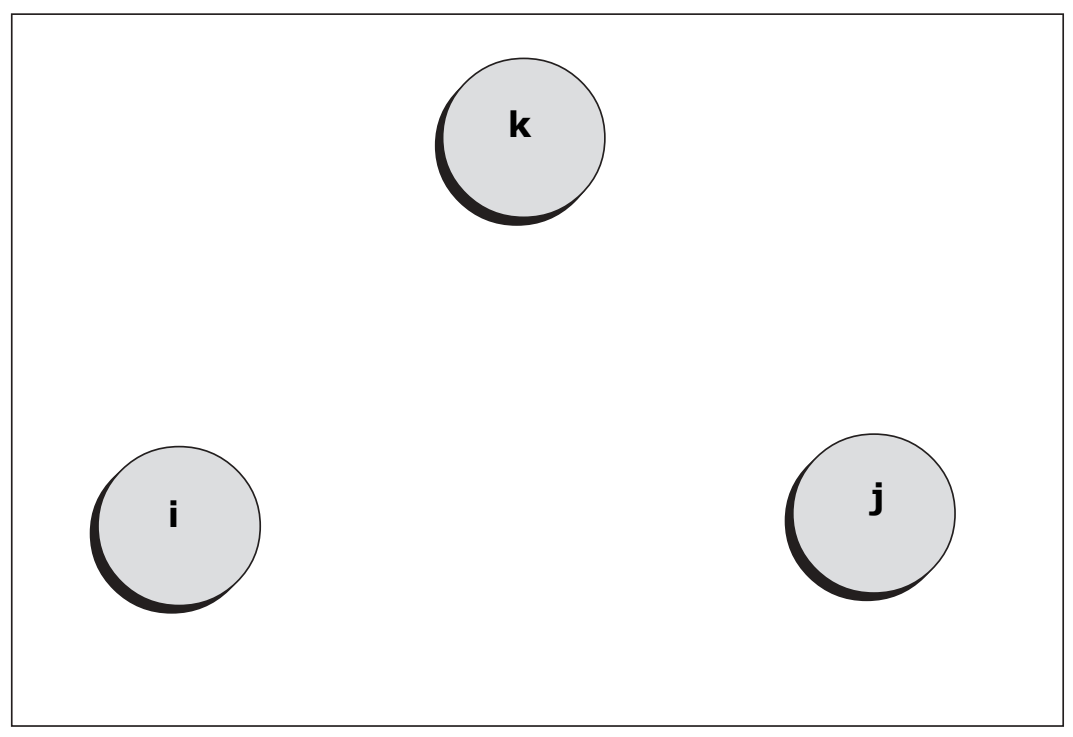

En este caso, cualquier cambio que afecte a la variable i puede repercutir sobre la variable j. Hay entonces una relación indirecta entre i y j. En la matriz de análisis estructural existen relaciones indirectas del tipo i-j que no pueden detectarse solo con la clasificación directa.

Al elevar al cuadrado la matriz, se pone en evidencia las relaciones de orden 2 entre $\boldsymbol{i}-\boldsymbol{j}$.
Al elevarla al cubo o a la cuarta potencia, se obtiene de la misma manera el número de caminos de influencia o bucles de influencia de orden 3 o 4, que unen las variables entre si. En cada repetición se deduce una nueva jerarquía de las variables, clasificadas esta vez en función del número de las acciones indirectas que ejercen sobre las demás variables.

Análisis estructural relaciones indirectas

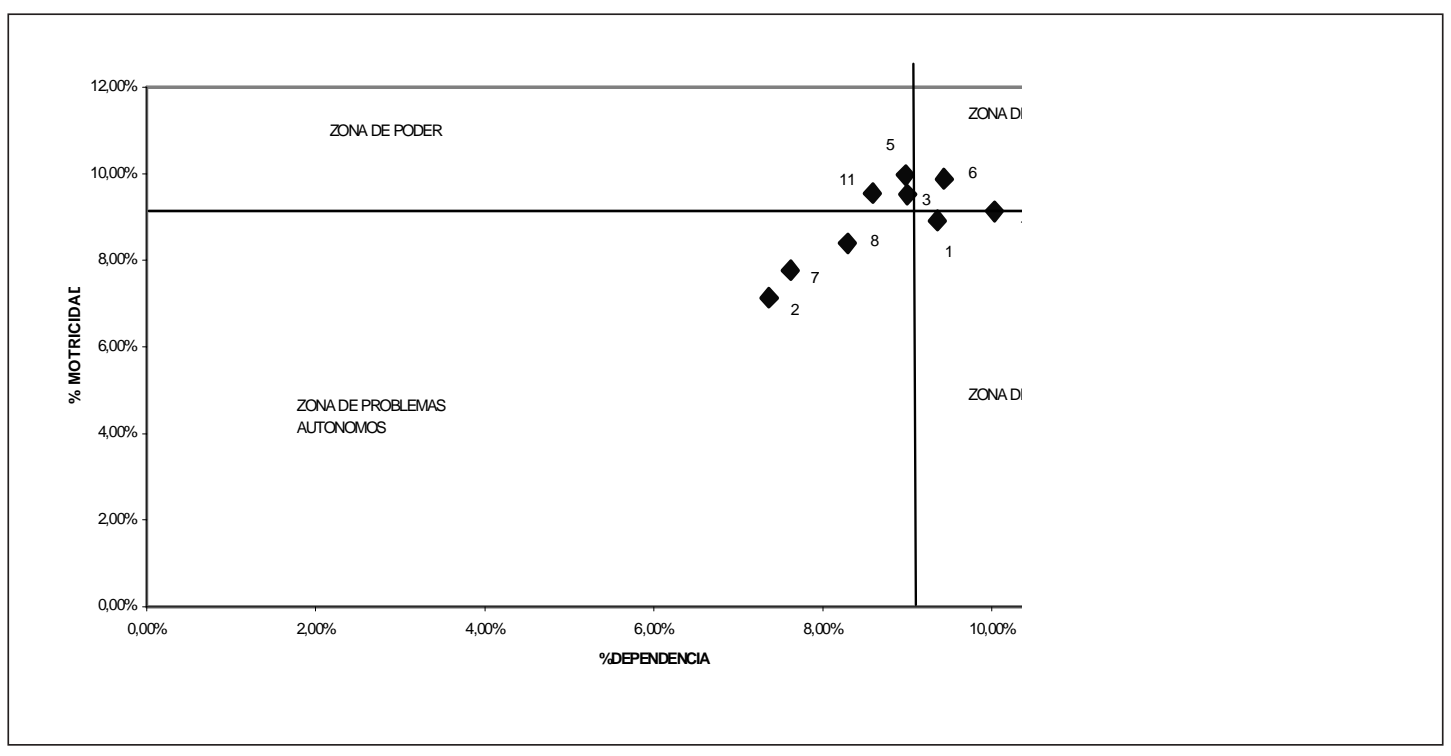




\section{Resultados del análisis estructural para identificar las relaciones indirectas entre las variables clave}

En este caso, el resultado señala que en la zona de poder (alta motricidad y baja dependencia) se encuentran las mismas variables de las relaciones directas. Asimismo, que en la zona de conflicto (alta motricidad y alta dependencia) están las mismas variables de las relaciones directas. Igual sucede para la zona de salida (alta dependencia y baja motricidad). En la zona de problemas autónomos (baja motricidad y baja dependencia) están las mismas variables de las relaciones directas.

La multiplicación matricial, aplicada a la matriz de análisis estructural antes descrita, permite afirmar que, en este caso, no se da una fuerte difusión de los impactos por los caminos y bucles de reacción y por consiguiente la jerarquía de las variables de motricidad (teniendo en cuenta el número de caminos y bucles salidos de cada variable) y de dependencia, o el número de caminos y bucles que llegan a cada variable, no se altera.

\section{ANÁLISIS DE RESULTADOS}

\section{Por dónde empezar}

La organización en red se plantea cómo llevar adelante un modelo de negocio. El modelo actual, informal, sin calidad, sin exigencias mínimas sobre los productos, no necesita una estructura en red. Cada cual se salva como puede.

Pero en una situación de competencia, incluso en el mercado interno, esta forma precaria de organización no es sostenible. Construir una nueva organización de los productores de hortalizas debe empezar por cambiar hacia un modelo de agro negocio; es decir, hacia una producción de hortalizas sanas, uniformes, controladas, con riego adecuado, con garantía de buenas prácticas en el proceso, listas para consumir; en fin: con un alto valor agregado.

Según los resultados alcanzados en los talleres de prospectiva, resulta conveniente empezar atendiendo las variables problema o de enlace (o zona de conflicto). Hay que recordar que estos factores son a la vez motrices y muy dependientes, inestables por naturaleza. Cualquier acción sobre estas variables repercutirá sobre las otras y tendrá un efecto bumerang sobre ellas mismas, lo que amplificará el impulso inicial.

En esta zona de conflicto, con alta motricidad y alta dependencia, están:

- Falta de incentivos y políticas públicas para que las empresas hagan alianzas, se asocien y atraigan inversión

- Reducida inversión pública y privada en ciencia, tecnología e innovación.

- Falta de inversión privada en acciones, capital de riesgo, fondos cerrados de inversión y acciones de banca de inversión.

- Difícil acceso al crédito dirigido y altos costos. 
Los llamados inversionistas estratégicos, es decir, las empresas que unen capital con los conocimientos específicos del negocio, pueden integrar estas variables. Tales inversionistas se diferencian de los inversionistas institucionales, en que estos son conserva-dores en esencia, pues buscan rentabilidad con poco riesgo. Aquellos, en cambio, buscan colocarse en el segmento del mercado que conocen y obtener una alta rentabilidad. Son empresarios con dinero y conocimientos del negocio. Capitanes de empresa que arriesgan recursos para contratar a futuro la producción de hortalizas de calidad, lo cual implica financiación y controles en todos los eslabones de la cadena de suministro del negocio. Hombres de negocio que generan la «destrucción creativa» de la que habla Schumpeter, como motor del cambio.

Un inversionista estratégico crea condiciones para que las empresas productoras de hortalizas hagan alianzas y se asocien; invierte en tecnología y en innovación; moviliza la inversión privada mediante emisión de acciones, bonos, BOCEAS -bonos convertibles en acciones-, forma fondos cerrados de inversión y actúa como banca de inversión, si se necesita. No es todopoderoso, pero conoce las condiciones del segmento del mercado y se mueve en él con fluidez, pues es su negocio.

Hay que atraerlos. La política pública departamental y municipal debe invertir para crear incentivos que disminuyan los riesgos inherentes a la operación; crear facilidades para acceder a mecanismos de la Bolsa Agropecuaria de Mercado de Futuros. Para crear garantías a la movilización regional de capitales.
Esta respuesta simple y de sentido común, es fácil de entender ahora. Cuando se inició el estudio, nadie la mencionaba. Ahora sí, y es parte de la contribución académica a entender una realidad para transformarla.

\section{Acciones derivadas}

Con una inversión estratégica como la mencionada se puede crear un sistema de información empresarial de precios, calidades, cantidades, desarrollos tecnológicos, novedades en productos, seguimiento a empresas internacionalizadas, conocimiento de políticas públicas favorables al segmento de mercado. Se puede empezar a construir el libro blanco sobre la horticultura de tierra fría, que es un espacio virtual organizado, en donde se envían contribuciones -previo análisis de un comité editorial-. Este recurso puede contribuir a fortalecer el conocimiento especializado sobre este tema.

Otro tema derivado es el de las mejoras en la asistencia técnica en mejores prácticas. En la medida en que los productores tengan un mayor control sobre el nivel de incertidumbre sobre el destino futuro de su producción, pueden concentrarse en lo que saben hacer: producir. A ello debe agregarse una mejora en productividad, procesos, manejos post cosecha y otros aspectos técnicos de los eslabones de las cadenas hortícolas.

Con una mejor información empresarial, una mayor asistencia técnica y un más claro domino de los niveles de incertidumbre sobre el destino de las cosechas, se puede generar confianza entre los participantes y el capital social. 


\section{Bibliografía}

CASSON, Mark (2000). Enterprise and Leadership: Studies on Firms, Markets and Networks. Northampton, MA, USA, Edgard Elgar.

CEPAL (1985). Los nuevos escenarios agrícolas en formación. LC/R.111. Santiago de Chile, 1985. La apertura y los procesos agroexportadores recientes. LC/R.1299, Santiago de Chile.

DNP (2005). Visión Colombia II Centenario, 2019: Propuesta para discusión. DNP, Planeta, Bogotá.

FUKUYAMA, Francis (1995). Trust: The Social Virtues and the Creation of Prosperity. New York, The Free Press.

GODET, Michel (1999). De la anticipación a la acción: manual de prospectiva y estrategia. México, Alfaomega.

GRANDORI, Anna (1999). Interfirm Networks: Organization and Industrial Competitiveness. Editor. London and New York, Routledge.

JARILLO, José Carlos (2003). Strategic Logic. New York, Palgrave Macmillan.
KRAMER, Roderick M.; and TYLER, Tom R. (1996). Trust in Organizations: Frontiers of Theory and Research. USA, Sage Publications.

KAPLAN, Robert S.; y NORTON, David P. (2001). The Strategy-Focused Organization. Boston, Harvard Business School Press.

PORTER, Michael (1980). Competitive Strategy: Techniques for Analyzing Industries and Competitors. New York, Free Press.

PORTER, Michael E. (1990). The Competitive Advantage of Nations. Harvard Business Review, March-april, Boston, USA.

PUTNAM, R.D.; LEONARDI, R.; and NANETTI, R.Y. (1993). Making Democracy Work: Civic Traditions in Modern Italy. Princeton, NJ, Princeton University Press.

PUTNAM, Robert D. (2002). Democracies in Flux: the Evolution of Social Capital in Contemporary Society. Editor. USA, Oxford University Press.

SANTOS, Milton (1995) «Los espacios de la globalización» Revista de la Universidad del Valle, abril de 1995, Número 10, páginas 36 a 41. 
